\title{
Relacionamento entre Filhos e Pais não Residentes, Representação de Apego e Desempenho de Escrita
}

\author{
Márcia Siqueira de Andrade \\ Centro Universitário FIEO \\ Alessandra Gotuzo Seabra \\ Universidade Presbiteriana Mackenzie

\section{Cristina Aparecida Lopes de Morais} \\ Centro Universitário FIEO
}

\begin{abstract}
RESUMO
Considerando que o apego interfere no desenvolvimento cognitivo pesquisou-se a relação entre filhos e pais não residentes, representação de apego, desempenho em escrita. Participaram 43 estudantes do Ensino Fundamental que não residiam com o pai. Utilizou-se para a coleta de dados: teste do desenho da família e avaliação das dificuldades de aprendizagem na escrita.Os resultados revelaram que o aumento dos sinais ansiosos/ inseguros correspondeu ao maior número de erros na escrita; quanto menor o tempo de não residência pai/filho, maior o número de erros na escrita. Os participantes que se relacionam com o pai tiveram melhor desempenho na escrita. Conclui-se que, tanto a representação de apego quanto a relação entre filhos e pais não residentes influenciam o desempenho na escrita.
\end{abstract}

Palavras-chave: Pais não residentes; representação de apego; escrita;

\section{ABSTRACT \\ Relationship between children and nonresident fathers, attachment representation and write performance}

Considering that the attachment interfere in cognitive development this research sought to understand the relationship between children and nonresident fathers, attachment representation and write performance. Attended by 43 elementary school students whose did not reside with their father being used for data collection: Family Drawing, Assessment of Learning Disabilities in Writing. Results showed increases in anxious and insecure signals corresponds to the highest number of errors in writing; the shorter the time non residence parent / child, the greater the number of errors in writing. Participants that relate to the father performed better in writing. This research concludes that both the attachment representation as the relationship between children and nonresident fathers influence the performance in writing.

Keywords: Nonresident fathers; attachment representation; writing.

Nas últimas décadas, a convivência paterna vem sendo estudada como um importante aspecto do papel do pai, verificando-se um gradual aumento nos seus níveis entre os pais que vivem com seus filhos (Rude-Antoine, 2009; Oliveira, 2010). No entanto, tem sido também observado um número crescente de pais que não residem com seus filhos, devido aos altos índices de divórcio de filhos fora do casamento (Cúnico, \& Arpini, 2013). No que se refere a estes pais, embora diversos estudos afirmem que eles costumam se afastar dos filhos ou desenvolver uma relação baseada unicamente em visitas e recreação (Rosa, 2003; Padilha, 2008; Cúnico, \& Arpi- ni, 2014), alguns deles demonstram ser capazes de manter suas funções como pai, preservando uma relação de proximidade com os filhos (Silva, 1999; Brito, 2003; Marsiglio, Amato, Day \& Lamb, 2001). Considera-se que o afastamento físico permanente pode predispor ao afastamento emocional (Sganzerla, \& Levandowski, 2010).

Conforme dados da Pesquisa Nacional por Amostra de Domicílios (Pnad) realizada pelo Instituto Brasileiro de Geografia e Estatística (IBGE) no ano de 2012, o padrão dominante do tipo de organização familiar ainda é a família nuclear (40,89\%), constituída pelo casal e seus 
filhos. Contudo, houve um expressivo crescimento relativo às famílias cuja responsabilidade é feminina, que são caracterizadas pelos laços entre mãe e criança, sem a presença de uma relação conjugal envolvendo coabitação ou com esta relação menos solidária e intensa afetivamente.

Vários fatores influenciam a aprendizagem da escrita pelas crianças (Resende, Oliveira, Godoy \& Denzin, 2010; Sitta, Arakawa, Caldana \& Peres, 2010). Dentre os fatores de maior relevância podem ser citados os orgânicos, os psicossociais e os cognitivos (Andrade \& Castanho, 2014). Entretanto, o interesse deste estudo é iluminar, nesse processo, aspectos da relação entre filhos e pais não residentes, uma vez que estudos sobre a relação entre pais e filhos têm mostrado a relevância da mãe nos cuidados com a prole e com sua socialização, isto é, na maternagem, mas não têm atribuído a mesma atenção à paternagem (Seben, 2010; Grzybowski, 2002).

Pesquisas focadas no pai e na paternidade aparecem em número reduzido nos meios científicos (Barbieri, 2009; Selan, 2009). Sganzerla e Levandwski (2010) apontam que estudos para a compreensão da relação entre pai e filho aparecem com mais constância a partir dos anos de 1980, período em que há um número crescente de separações e divórcios, evidenciando a importância do pai para o desenvolvimento da criança.

Entretanto, grande parte desses estudos foi conduzida em contextos culturais diferentes do brasileiro, o que não permite saber como a relação entre filhos e pais não residentes interfere no desenvolvimento dos filhos no Brasil (Régnier-Loilier, 2015; Giardini, 2014; Schober, 2012; Hook \& Wolfe, 2012). Além disso, o papel do pai se encontra em uma fase de transição, sendo frequentes as famílias em que ambos os genitores estão inseridos no mercado de trabalho, o que tem levado à redefinição do papel paterno, com algumas das responsabilidades familiares sendo repassadas aos homens, principalmente em relação aos cuidados com os filhos (Lewis \& Dessen, 2012; Bruschini \& Ricoldi, 2012). Os impactos dessas mudanças no desenvolvimento infantil somente poderão ser conhecidos a partir de estudos que avaliem a interação entre pai e filho e o desenvolvimento infantil. Estudos dessa natureza ajudariam ainda na identificação de fatores de risco, o que é importante, considerando-se o contexto da educação brasileira, em que há um número cada vez maior de alunos com baixo desempenho acadêmico.
Nesse contexto, o objetivo do presente estudo foi investigar crianças do terceiro ao quinto ano do Ensino Fundamental I, que não residiam com os pais, analisando a relação entre representação de apego, tempo e tipo de não residência pai/filho (morte, abandono ou separação conjugal) e desempenho escolar na escrita. São chamados pais não residentes aqueles que não moram com os filhos em decorrência do abandono, falecimento ou afastamento pelo divórcio (Silva \& Piccinini, 2004). Entende-se, ainda, neste estudo, apego como um mecanismo básico dos seres humanos que funciona dentro de um contexto de outros sistemas de controle comportamentais. O papel do apego na vida dos seres humanos envolve o conhecimento de que uma figura de apego está disponível e oferece respostas, proporcionando um sentimento de segurança que é fortificador da relação (Cassidy, 1999).

\section{MÉTODO}

Para atingir os objetivos propostos foi realizado estudo com delineamento correlacional e caráter transversal.

\section{Participantes}

Foram indicados pela equipe escolar 50 alunos que atendiam aos seguintes critérios de inclusão: presença de queixa do aluno ou da família sobre a ausência paterna; matrícula no terceiro, quarto ou quinto ano do Ensino Fundamental I na ocasião da pesquisa; não apresentação de necessidades especiais comprovadas por laudo clínico; presença constante da mãe ou moradia em comum com ela; e não coabitação com o pai, cuja convivência com o participante poderia ser: frequente, com participação semanal; esporádica, ou seja, presente algumas vezes por ano; ou convívio inexistente.

Dentre os 50 alunos indicados pela equipe escolar, 43 deles foram autorizados pelos responsáveis a participar do estudo, por meio de Termo de Consentimento Livre e Esclarecido. A tabela 1 apresenta a análise descritiva em relação às variáveis idade/escolaridade/sexo dos participantes.

A relação idade/escolaridade/sexo apresenta-se da seguinte forma:

a) seis meninas e dois meninos com oito anos de idade, no quarto ano, nenhum deles com defasagem idadelano escolar; 
b) uma menina e sete meninos no quarto ano e uma menina no quinto ano, todos com nove anos;

c) com dez anos, 13 meninas (nove delas no quinto ano e quatro no quarto ano) e sete meninos (um no quarto ano e seis no quinto ano);

d) seis alunos com 11 anos: quatro meninas no quinto ano, uma no quarto ano e dois meninos no quinto ano.
Constata-se que 11 alunos participantes apresentam defasagem idade/escolaridade (24,5\%), sendo seis deles no quarto ano e cinco no quinto ano.

\section{Instrumentos}

Os instrumentos utilizados para a coleta de dados foram: prontuário escolar do participante, entrevista semiestruturada realizada com a mãe ou responsável, teste do desenho da família (TDF) e avaliação das dificuldades de aprendizagem na escrita (ADAPE),

Tabela 1

Idade/escolaridade/sexo dos participantes

\begin{tabular}{|c|c|c|c|c|c|c|c|c|c|c|}
\hline \multirow{2}{*}{ Escolaridade } & \multicolumn{2}{|c|}{8 anos } & \multicolumn{2}{|c|}{9 anos } & \multicolumn{2}{|c|}{10 anos } & \multicolumn{2}{|c|}{11 anos } & \multicolumn{2}{|c|}{ Total } \\
\hline & Fem & Masc & Fem & Masc & Fem & Masc & Fem & Masc & Fem & Masc \\
\hline $3^{\circ}$ ano & 6 & 2 & 0 & 0 & 0 & 0 & 0 & 0 & 6 & 2 \\
\hline $4^{\circ}$ ano & 0 & 0 & 1 & 7 & 4 & 1 & 1 & 0 & 6 & 8 \\
\hline $5^{\circ}$ ano & 0 & 0 & 1 & 0 & 9 & 6 & 3 & 2 & 13 & 8 \\
\hline Total & 6 & 2 & 2 & 7 & 13 & 7 & 4 & 2 & 25 & 18 \\
\hline
\end{tabular}

Nota: Dados retirados do prontuário escolar

proposta por Sisto (2001). Os dois últimos encontramse descritos a seguir.

A versão do TDF utilizada foi a proposta por Fury, Carlson e Sroufe (1997), validada no Brasil por Cecconello, Krum e Koller (2000). O teste é composto de duas escalas: escala de frequência de sinais específicos e escala global. A primeira avalia sinais específicos presentes no desenho e é composta de 24 itens que descrevem características do desenho. Desenhos que apresentam determinado sinal recebem um ponto; desenhos que não apresentam o sinal recebem zero ponto. $\mathrm{O}$ valor total pode chegar a 24 pontos. A maior pontuação indicará maior comprometimento afetivo

A segunda escala, a escala global, objetiva avaliar o desenho como um todo e é composta de oito subescalas: vitalidade/criatividade, felicidade/orgulho da família, vulnerabilidade, distância emocional/isolamento, tensão/raiva, papéis invertidos, dissociação e patologia global. As escalas distância emocional/isolamento e tensão/raiva estão relacionadas com histórias de apego ansioso evitante; a escala vulnerabilidade, com apego ansioso resistente; a escala papéis invertidos está relacionada com histórias de apego ansioso resistente; as escalas vitalidade/criatividade e felicidade/orgulho da família estão relacionadas com apego inseguro de maneira geral; e a escala patologia global está relacionada com os três tipos de apego: evitante, resistente e inseguro. Na escala global, a pontuação pode variar de 7 a 1 , que correspondem, respectivamente, a muito alto, alto, moderadamente alto, moderado, moderadamente baixo, baixo e muito baixo.

Para efeito de análise, serão consideradas nesta pesquisa as oito subescalas da escala de frequência de sinais específicos, uma vez que avaliam aspectos específicos do desenho, e a subescala patologia global, da escala global, considerando que esta se relaciona aos três tipos de apego e está designada para captar o grau total de patologia refletida no desenho.

Os desenhos foram analisados, individualmente, por dois avaliadores, que se reuniram, posteriormente, para discutir eventuais discordâncias, até obter consenso. Quando discordâncias posteriores aconteciam, um terceiro avaliador intervinha no processo e participava da discussão até obter consenso.

A ADAPE (Sisto, 2001) foi utilizada para avaliar as dificuldades de aprendizagem da escrita, que se diferenciam a depender da escolaridade dos alunos. Avalia especificamente as dificuldades relativas à representação de fonemas, não avaliando aspectos referentes à compreensão e à expressão de significados, e possibilita a identificação do nível de alfabetização. A 
ADAPE é constituído por um ditado composto de 114 palavras. De forma geral, cada palavra é considerada uma unidade. Cada unidade errada recebe o valor $1 \mathrm{e}$ cada unidade correta recebe 0 . Assim, a soma dos erros fornece a pontuação de cada participante. Além disso, a escala avalia também o uso correto de parágrafos, letra maiúscula e acentuação.

\section{Procedimentos}

A coleta de dados foi realizada em uma escola da rede municipal de Itapecerica da Serra que atende 450 alunos do Ensino Fundamental I (primeiro ao quinto ano). Foi realizado o levantamento dos dados dos 43 participantes em documentos escolares. Em uma nova data, grupos de participantes (dez a 11) foram conduzidos para uma sala disponibilizada para a aplicação da ADAPE (Sisto, 2001). Na sala, a pesquisadora informou aos participantes que faria um ditado, que as palavras seriam lidas uma a uma e não seriam repetidas. Foram disponibilizados a cada participante uma folha de papel sulfite tamanho A4, lápis preto número 2 e borracha. Iniciou-se o ditado, com duração de aproximadamente 45 minutos.

Por fim, solicitou-se aos participantes que, individualmente, desenhassem uma família. Para o desenho, foram disponibilizados uma folha de papel sulfite tamanho $\mathrm{A} 4$, lápis preto número 2, lápis de cor, borracha, régua e canetas do tipo hidrocor, os quais poderiam ser usados livremente. Após a finalização do desenho, solicitou-se aos participantes que informassem a existência de pai ou mãe no desenho.

\section{Considerações éticas}

A pesquisa foi aprovada pelo Comitê de Ética em Pesquisa da instituição promotora, Centro Universitário FIEO, sob o protocolo 037/2011. Atendendo à Resolução 196/96 do Conselho Nacional de Saúde, foi emitida carta para informar aos responsáveis pelos participantes o objetivo do estudo e assegurar que as informações seriam tratadas de forma anônima e sigilosa e serviriam apenas para fins técnico-científicos. Foi ainda disponibilizada assistência, caso algum participante necessitasse de apoio psicológico (Resolução 196/96 do Conselho Nacional de Saúde).

\section{RESULTADOS}

O Statistical Package for Social Sciences - SPSS v. 17.0 foi utilizado para a construção do banco de dados e a análise das informações. Os dados provenientes da avaliação do TDF, da ADAPE e das entrevistas foram inicialmente analisados de forma descritiva. Para verificar as possíveis relações entre desempenho na ADAPE e sinais no TDF, foi conduzida análise de correlação linear de Pearson. A tabela 2 apresenta os coeficientes da correlação entre a ADAPE e as cinco medidas de sinais do TDF: os quatro sinais específicos (sinais ansiosos ou evitantes, sinais ansiosos ou

Tabela 2

Análise da correlação de Pearson entre os fatores do ADAPE e do TDF

\begin{tabular}{lcc}
\hline Fatores do TDF & $\boldsymbol{r}$ & $\boldsymbol{p}$ \\
\hline Sinais Ansiosos Evitantes & 0,23 & 0,140 \\
Sinais Ansiosos Inseguros & $0,31^{*}$ & 0,041 \\
Sinais Ansiosos Resistentes & 0,02 & 0,888 \\
Sinais Desorganizados/Desorientados & $-0,16$ & 0,315 \\
Patologia Global & 0,16 & 0,311 \\
\hline
\end{tabular}

inseguros, sinais ansiosos ou resistentes e sinais organizados/desorganizados) e a escala global (Fury, Carlson, \& Sroufe, 1997).

Observa-se correlação significativa entre os fatores da ADAPE e uma das dimensões do TDF, os sinais ansiosos e inseguros. O coeficiente foi positivo e de magnitude baixa $(r=0,31 ; p=0,041)$. Assim, esse resultado sugere que, à medida que os sinais ansiosos $\mathrm{e}$ inseguros aumentam, há uma tendência de o número de erros na escrita também aumentar.

Para verificar se o desempenho na ADAPE poderia diferir em função do tempo de não residência com os pais, foi conduzida análise de correlação de Pearson. Os resultados indicam que não houve correlação significativa, embora tenha sido identificada uma tendência negativa $(r=-0,272 ; p=0,083)$, ou seja, quanto menor o tempo de não residência pai/filho, maior o número de erros na escrita. Esse resultado, apesar de não significativo, pode sugerir que o impacto da não residência pai/filho sobre o desempenho na escrita seja amenizado com o tempo e, possivelmente, com o surgimento de outros substitutivos significativos.

Para verificar se a representação mental de apego diferia em função do tempo de não residência pai/filho, realizou-se análise de correlação de Pearson entre os indicadores do TDF e o tempo de não residência 
pai/filho.

Tabela 3

Análise da correlação de Pearson entre TDF e tempo de não residência pai/filho $(N=43)$

\begin{tabular}{lcc}
\hline Fatores do TDF & $r$ & $p$ \\
\hline Sinais Ansiosos Evitantes & 0,180 & 0,500 \\
Sinais Ansiosos Inseguros & 0,109 & 0,498 \\
Sinais Ansiosos Resistentes & $-0,035$ & 0,828 \\
Sinais Desorganizados/Desorientados & 0,089 & 0,580 \\
Patologia Global & $-0,008$ & 0,959 \\
\hline
\end{tabular}

Os resultados indicam que não há relação significativa entre os escores do TDF e o tempo de não residência pai/filho. Pode-se aferir que a representação mental de apego não se relacionou com o tempo de não residência pai/filho.

Para comparar os resultados na ADAPE e no TDF quanto ao motivo da não residência pai/filho, foram considerados dois grupos: o primeiro grupo representa os participantes cujos pais separaram-se das mães ou as abandonaram ( $\mathrm{f}=88,3 \%$ ); o segundo grupo representa os participantes cujos pais faleceram $(\mathrm{f}=11,7 \%)$.

Tendo em vista a distribuição dos dois grupos considerados, foi necessário analisar a homogeneidade $\mathrm{e}$ a normalidade das variâncias. Assim, utilizaram-se o teste Levene e o teste Kolmogorov-Smirnov, que confirmaram o pressuposto da normalidade e da homogeneidade das variâncias. Dessa forma, foi conduzida análise de variância multivariada na comparação das medidas. A tabela 4 apresenta os resultados da análise.

Tabela 4

\begin{tabular}{|c|c|c|c|c|c|}
\hline & Motivo da não residência & Média & Desvio padrão & $f(1,41)$ & $p$ \\
\hline \multirow{3}{*}{ ADAPE } & Abandono & 29,03 & 23,71 & 0,79 & 0,379 \\
\hline & Falecimento & 39,80 & 38,01 & & \\
\hline & Total & 30,28 & 25,39 & & \\
\hline \multirow{4}{*}{ Sinais Ansiosos/Evitantes } & Abandono & 0,21 & 0,17 & 3,87 & 0,056 \\
\hline & Falecimento & 0,06 & 0,08 & & \\
\hline & Total & 0,20 & 0,17 & & \\
\hline & Abandono & 0,20 & 0,21 & 0,01 & 0,979 \\
\hline \multirow[t]{2}{*}{ Sinais Ansiosos/ Inseguros } & Falecimento & 0,20 & 0,22 & & \\
\hline & Total & 0,20 & 0,21 & & \\
\hline \multirow{3}{*}{$\begin{array}{l}\text { Sinais Ansiosos/ } \\
\text { Resistentes }\end{array}$} & Abandono & 0,14 & 0,13 & 0,30 & 0,587 \\
\hline & Falecimento & 0,18 & 0,07 & & \\
\hline & Total & 0,15 & 0,13 & & \\
\hline \multirow{3}{*}{$\begin{array}{l}\text { Sinais Desorganizados/ } \\
\text { Desorientados }\end{array}$} & Abandono & 0,04 & 0,18 & 0,30 & 0,585 \\
\hline & Falecimento & 0,00 & 0,00 & & \\
\hline & Total & 0,04 & 0,17 & & \\
\hline \multirow{3}{*}{ Patologia Global } & Abandono & 26,45 & 5,62 & 0,16 & 0,689 \\
\hline & Falecimento & 25,40 & 3,78 & & \\
\hline & Total & 26,33 & 5,41 & & \\
\hline
\end{tabular}

Os resultados sugerem que o motivo de não residência pai/filho não interferiu de forma significativa no desempenho na ADAPE ou no TDF, ou seja, em nenhuma das medidas houve diferença entre os grupos, sendo indiferente a não residência pai/filho ocorrer por falecimento ou por abandono/separação do pai.

Apenas no escore de sinais ansiosos/evitantes do TDF houve um efeito marginal $(p=0,056)$, sendo que o grupo de crianças cujos pais abandonaram a família 
Tabela 5

Comparação das medidas do TDF e do ADAPE entre grupos divididos quanto à frequência da visitação do pai

\begin{tabular}{|c|c|c|c|c|c|c|}
\hline & Frequência da visita & Média & Desvio Padrão & $\mathrm{N}$ & $f(3,39)$ & $\bar{p}$ \\
\hline \multirow{5}{*}{ ADAPE } & Frequente & 16,00 & 7,71 & 5 & 2,84 & 0,050 \\
\hline & Esporádica & 16,29 & 7,78 & 7 & & \\
\hline & Não vê & 40,62 & 31,51 & 21 & & \\
\hline & Não conheceu & 25,50 & 14,02 & 10 & & \\
\hline & Total & 30,28 & 25,39 & 43 & & \\
\hline \multirow{5}{*}{ Sinais Ansiosos/ Evitantes } & Frequente & 0,14 & 0,10 & 5 & 0,19 & 0,905 \\
\hline & Esporádica & 0,20 & 0,20 & 7 & & \\
\hline & Não vê & 0,20 & 0,18 & 21 & & \\
\hline & Não conheceu & 0,21 & 0,18 & 10 & & \\
\hline & Total & 0,20 & 0,17 & 43 & & \\
\hline \multirow{5}{*}{ Sinais Ansiosos/ Inseguros } & Frequente & 0,13 & 0,14 & 5 & 0,76 & 0,522 \\
\hline & Esporádica & 0,29 & 0,23 & 7 & & \\
\hline & Não vê & 0,21 & 0,22 & 21 & & \\
\hline & Não conheceu & 0,15 & 0,18 & 10 & & \\
\hline & Total & 0,20 & 0,21 & 43 & & \\
\hline \multirow{5}{*}{ Sinais Ansiosos/Resistentes } & Esporádica & 0,21 & 0,16 & 7 & 0,94 & 0,430 \\
\hline & Frequente & 0,13 & 0,09 & 5 & & \\
\hline & Não conheceu & 0,11 & 0,16 & 10 & & \\
\hline & Não vê & 0,14 & 0,11 & 21 & & \\
\hline & Total & 0,15 & 0,13 & 43 & & \\
\hline \multirow{5}{*}{$\begin{array}{l}\text { Sinais Desorganizados } \\
\text { /Desorientados }\end{array}$} & Frequente & 0,00 & 0,00 & 5 & 0,54 & 0,655 \\
\hline & Esporádica & 0,10 & 0,16 & 7 & & \\
\hline & Não vê & 0,05 & 0,22 & 21 & & \\
\hline & Não conheceu & 0,00 & 0,00 & 10 & & \\
\hline & Total & 0,04 & 0,17 & 43 & & \\
\hline \multirow{5}{*}{ Patologia Global } & Frequente & 27,00 & 3,24 & 5 & 0,32 & 0,808 \\
\hline & Esporádica & 27,86 & 5,21 & 7 & & \\
\hline & Não vê & 26,14 & 6,00 & 21 & & \\
\hline & Não conheceu & 25,30 & 5,54 & 10 & & \\
\hline & Total & 26,33 & 5,42 & 43 & & \\
\hline
\end{tabular}

apresentou tendência a maior frequência desse sinal do que o grupo de crianças cujos pais faleceram.

Para comparar os desempenhos na ADAPE e no TDF em função da frequência de visitação do pai, foi conduzida análise de variância, com os resultados apresentados na tabela 5 .

Observa-se efeito significativo da frequência de visitação do pai somente sobre o escore da ADAPE. Nesse teste, o grupo de crianças que não vê o pai foi o que apresentou os piores desempenhos, com maior número de erros na escrita. O segundo pior desempenho na ADAPE foi do grupo de participantes que não conheceram o pai. Os participantes que veem os pais esporádica ou frequentemente tiveram os melhores desempenhos. Já no TDF, não houve diferença significativa entre os participantes com diferentes frequências de contato com os pais. Os resultados sugerem, portanto, que as crianças com melhor desempenho na escrita foram as que conviviam mais com o pai.

\section{DISCUSSÃO}

Os resultados desta pesquisa revelaram que, conforme os escores dos sinais ansiosos e inseguros do TDF aumentam, o número de erros na escrita também 
cresce. Estudos realizados nos últimos anos apoiam a hipótese de que a alta ansiedade interfere negativamente na performance acadêmica (Torunsky \& Lopes, 2012). Ainda sobre essa questão, Yaegashi (1997) menciona que, em estudos sobre os fatores emocionais e sua relação com a aprendizagem, constatou-se que os problemas emocionais podem, de fato, influenciar o rendimento escolar, principalmente no início da escolarização - caso dos participantes do presente estudo.

Pesquisa realizada por Jacob, Loureiro, Marturano, Linhares e Machado (1999) sobre a relação entre problemas de aprendizagem e aspectos afetivos verificou que crianças com queixa de atraso escolar tiveram maior dificuldade para a utilização dos recursos potenciais intelectuais e afetivos, caracterizando empobrecimento, menor capacidade de elaboração e imaturidade. Nesse sentido, para aqueles autores, o rendimento escolar, em geral considerado um sinal adaptativo importante, mostrou-se acompanhado de dificuldades afetivas.

Para Bazi (2000), a ansiedade se caracteriza como um problema emocional que interfere no aprendizado da escrita, principalmente porque tal afeto se relaciona a uma grande confusão mental, o que pode influenciar a representação gráfica de um determinado conteúdo. Para essa autora (Bazi, 2000), quanto maior o nível de ansiedade, pior o desempenho na leitura e na escrita, o que corrobora os resultados encontrados neste estudo.

Os resultados indicam, também, que não há correlação linear entre os escores da ADAPE ou do TDF e o motivo da não residência pai/filho, seja falecimento, seja abandono. Esses resultados diferem dos apresentados na literatura. Pinheiro, Siqueira e Bucher (1983) afirmam que as consequências da ausência paterna assumem características distintas conforme a ausência do pai se dê por morte, abandono, separação ou divórcio. Essa questão parece ser relativizada pela forma como a mãe lida com a perda do companheiro.

Verificou-se, também, que, quanto menor o tempo de não residência pai/fillho, maior o número de erros na escrita. Esse resultado indica que o impacto da separação do pai no desempenho na escrita é amenizado com o tempo, sugerindo que a ausência paterna pode ser compensada pela dedicação da mãe ou por outros fatores, como o apoio social de avós e parentes, o que pode substituir, ao menos parcialmente, o papel do pai (Burchinal, Roberts, Nabors, \& Bryant, 1996). Sobre essa questão, Santos e Navas (2004), ao estudarem os efeitos da ausência paterna na aprendizagem de adultos cujas infâncias foram marcadas pela falta do pai, concluíram que essa demanda não repercutiu negativamente nos participantes, que, com o tempo, utilizaram outros substitutivos significativos.

Observa-se ainda que as crianças do grupo que não convive com o pai foram as que apresentaram maior número de erros na escrita, com desempenho inferior ao do grupo que não conheceu o pai. Os melhores escores da ADAPE foram daqueles que veem o pai esporádica e frequentemente. Esses resultados confirmam estudos de Biller e Kimpton (1997) e De Campos (2010), que concluíram que a ausência paterna para crianças em idade escolar é um fator de risco para desenvolvimento de problemas psicológicos, frequência de interações sociais e competência social e intelectual.

Cia, D'Affonseca e Barham (2004), ao estudarem o contexto brasileiro, confirmaram que, quanto mais frequentes as interações entre os pais e seus filhos, melhor o desempenho acadêmico das crianças, corroborando pesquisas realizadas em outros contextos culturais que também demonstraram a importância da qualidade do relacionamento entre pai e filho para o desempenho acadêmico das crianças (Flouri \& Buchanan, 2003; Pelegrina, García-Linares, \& Casanova, 2003).

\section{Considerações Finais}

Pesquisou-se a relação entre filhos e pais não residentes, representação de apego e desempenho escolar em escrita. Os resultados revelaram que, conforme os sinais de ansiedade e insegurança aumentam, o número de erros na escrita também cresce. Verificou-se, também, que, quanto menor o tempo de não residência pai/filho, maior o número de erros na escrita, sugerindo que, com o tempo, o impacto da separação do pai no desempenho na escrita é amenizado. Além disso, os participantes que se relacionam com o pai esporádica ou frequentemente tiveram melhor desempenho na escrita Os participantes que não veem os pais tiveram desempenhos piores do que aqueles que não conheceram os pais.

Tais resultados permitem afirmar que tanto a representação mental de apego quanto a relação entre 
filhos e pais não residentes influenciam o desempenho na escrita dos alunos do Ensino Fundamental.

Embora os objetivos tenham sido alcançados, algumas limitações desta pesquisa podem ser consideradas para novos estudos. Dentre as possibilidades, destacam-se:

a) a importância de se realizar uma distinção entre os efeitos diretos da ausência paterna e os indiretos, tais como aqueles advindos de mudanças no comportamento da mãe;

b) a importância de se coletarem os dados diretamente com o pai não residente sobre sua relação com o filho;

c) ampliação do número de participantes variando o contexto escolar e econômico de forma a favorecer generalizações e ampliar o poder estatístico dos testes empregados;

d) a importância da realização de estudos longitudinais, para verificar as contribuições que a figura paterna oferece ao longo das diferentes etapas do desenvolvimento infantil.

Por fim, espera-se que este estudo possa incitar o desenvolvimento de pesquisas sobre o tema.

\section{REFERÊNCIAS}

Ainsworth, M. (1989) Attachments beyond the infancy. American Psychologist, 44 (4), 709- 716.

Andrade, M.S. , \& Castanho, M. I. S. (2014). Caracterização do perfil de clientela de clínica-escola de psicopedagogia. Revista Psicopedagogia, 31(95), 101-108.

Barbieri, V. (2009). A influência da personalidade materna e paterna na etiologia da tendência antissocial. In S. N. Jesus, M. M. Rezende, \& I. Leal (Orgs.), Experiências e intervenções em psicologia da saúde, (pp. 1183-1198). Faro, Portugal: Universidade do Algarve.

Barbieri, V., \& de Godoy Pavelqueires, J. (2012). Personalidade Paterna como Fator Prognóstico no Tratamento da Tendência Antissocial1. Paidéia, 22(51), 101-110.

Bazi, G.A.P. (2000). As dificuldades de aprendizagem em leitura e escrita e suas relações com a ansiedade (Dissertação de mestrado). Programa de Pós-Graduação em Educação, Universidade Estadual de Campinas, São Paulo.

Brito, L. M. T. (2003). Igualdade e divisão de responsabilidades: pressupostos e conseqüências da guarda conjunta. In G. C. Groeninga \& R. C. Pereira (Orgs.), Direito de família e psicanálise: rumo a uma nova epistemologia (pp. 325-337). Rio de Janeiro: Imago.
Bruschini, M. C. A., \& Ricoldi, A. M. (2012). Revendo estereótipos: o papel evendo estereótipos: o papel dos homens no trabalho doméstico. Estudos Feministas, 20(1), 259-287.

Burchinal, M. R., Roberts, J. E., Nabors, L. A., \& Bryant, D. M. (1996). Quality of center child care and infant cognitive and language development. Child Development, 67, 606-620.

Cecconello, A. M., Krum, F. M., \& Koller, S. (2000). Indicadores de risco e proteção no relacionamento mãe-criança e representação mental da relação de apego. Psico, $31,81-122$.

Cia, F., \& Barham, J. (2009). O envolvimento paterno e o desenvolvimento social de crianças iniciando as atividades escolares. Psicologia em Estudo, 14 (1), 67-74.

Cia, F., D’Affonseca, S. M. \& Barham, E. J. (2004). A relação entre envolvimento paterno e desempenho acadêmico dos filhos. Cadernos de Psicologia e Educação Paidéia, 14(29), 277-286.

Cúnico, S. D., \& Arpini, D. M. (2013). O afastamento paterno após o fim de um relacionamento amoroso: um estudo qualitativo. Interação Psicol., 17 (1), 99-108. http://dx.doi.org/10.5380/psi.v17i1.27560.

Cúnico, S. D., \& Arpini, D. M. (2014). Não basta gerar, tem que participar?: um estudo sobre a ausência paterna. Psicologia: Ciência e Profissão, 34(1), 226-241. http://dx.doi.org/10.1590/S1414-98932014000100016

De Campos, M. T. (2010). Ausência paterna e suas repercussões sobre o desenvolvimento infantil (Dissertação de mestrado). Programa de Pós-Graduação em Psicologia da Saúde, Universidade Metodista de São Paulo, São Bernardo do Campo.

Feldman, R. (2000). Parents' convergence on sharing and marital satisfaction, father involvement, and parent-child relationship at the transition to parenthood. Infant Mental Health Journa1, 21, 176-191.

Fury, G., Carlson, E. A. \& Sroufe, L. A. (1997). Children's representations of attachment relationships in family drawings. Child Development, 68, 1154-1164.

Giardini, F. (2014). Joint Custody of Children on Separation and Divorce: The Current Law in Italy: An Overview of the Law and How It Is Applied. Int'l Surv. Fam. L., 227.

Grzybowski, L. S. (2002). Famílias monoparentais - mulheres divorciadas chefes de família. In A. Wagner (Org.), Família em cena - tramas, dramas e transformações (pp. 39-53). Petrópolis, RJ: Vozes.

Grzybowski, L. S., \& Wagner, A. (2010). Casa do pai, casa da mãe: a coparentalidade após o divórcio. Psicologia: Teoria e Pesquisa, 26(1), 77-87.

Hook, J. L., Wolfe, C. M. (2012). New Fathers? Residential Fathers' Time With Children in Four Countries. Journal of Family Issues, 33(4), 415- 450.

Instituto Brasileiro de Geografia e Estatística (2012). Pesquisa nacional por amostra de domicílios. Recuperado 
em 10 de março de 2013, de http://www.ibge.gov.br.

Jacob, A.V.; Loureiro, S.R.; Marturano, E.M.; Linhares, M.B.M. \& Machado, V.L.S (1999). Aspectos afetivos e o desempenho acadêmico de escolares. Psic.: Teor. e Pesq. $15(2), 153-162$.

Levandowski, D. C., Piccinini, C. A., \& Lopes, R. D. C. S. (2009). O processo de separação-individuação em adolescentes do sexo masculino na transição para a paternidade. Psicologia: Reflexão e Crítica, 22(3), 353-361.

Lewis, C., \& Dessen, M. A. (2012). O pai no contexto familiar. Psicologia: teoria e pesquisa, 15(1), 009-016.

MacLanaham, S. \& Teitler, J. (1999). The consequences of father absence. In M. Lamb (Org.). Parenting and child development in nontraditional family. (pp.83-102). New Jersey: Lawrence Herbaum Associates.

Marsiglio, W., Amato, P., Day, R. \& Lamb, M. (2001). Scholarship on fatherhood in the 1990s and beyond. Em R. M. Milardo (Org.), Understanding families into the new millennium: A decade in review. National Council on Family Relations (pp.392-410). Minneapolis: Minnesota.

Oliveira, E.T., (2010). Pai, separado e pobre: Entre as dificuldades e o desejo de uma paternidade plena. Dissertação de Mestrado Não-Publicada, Programa de Pós-graduação em Psicologia, Universidade Federal de Pernambuco, Pernambuco.

Pelegrina, S., García-Linares, M. C. \& Casanova, P. F. (2003). Adolescents and their parents' perceptions about parenting characteristics. Who can better predict the adolescent's academic competence? Journal of Adolescence, 26, 651-665.

Pinheiro,A.; Siqueira I.L. \& Bucher, J. (1983). Ausência do pai. Uma introdução ao tema. Revista de Psicologia, 1(1), $107-122$

Régnier-Loilier, A. (2015). When Fathers Lose Touch with Their Children After Separation. In The Contemporary Family in France (pp. 139-157). Springer International Publishing.

Resende, D. B. D., Oliveira, R. N. G. F. D., Godoy, A. C. D. S., \& Denzin, S. S. (2010). Intervenção lúdico-pedagógica: promovendo aprendizado em crianças com defasagem na lecto-escrita. Anuário da Produção de Iniciação Científica Discente, 10(11), 172-184.

Resolução No 196, de 10 de outubro de 1996. (1996, 10 de outubro). Diretrizes e normas regulamentadoras de pesquisas envolvendo seres humanos. Brasília, DF: Ministério da Saúde, Conselho Nacional de Saúde. Recuperado em 20 de março de 2012 de http://www.datasus.gov.br/conselho/reso196/RES19696.htm.

Rocha R.F. \& Oliveira G. M. (2008). Paternidade sócio- afetiva: $\mathrm{O}$ afeto faz apelo à paternidade. IBDFAM Acadêmico.

Rude-Antoine, E. (2009). A ruptura intergeracional em situação migratória. Questão de autoridade, olhar dos filhos sobre seu pai e sobre a paternidade. Psicologia em revis- ta, 15(2), 1-16.

Rutter, M. (1972). Maternal deprivation. Baltimore: Penguin Books.

Santos, M.T.M., \& Navas, A.L.G.P. (2004) Distúrbios da leitura e escrita. São Paulo: Manole.

Schober, P. S. (2012). Paternal child care and relationship quality: A longitudinal analysis of reciprocal associations. Journal of Marriage and Family, 74(2), 281-296.

Seben, G. (2010). A família em transformação: aspectos psicossociais da criança em duas distintas configurações familiares (Dissertação de mestrado). Programa de Pós-graduação em Psicologia, Universidade Federal do Rio Grande do Sul, Rio Grande do Sul.

Selan, B. (2009). Psicodinamismos de pais de crianças com tendência antissocial (Monografia de iniciação científica). Universidade de São Paulo, Ribeirão Preto-SP.

Sganzerla, I.M., \& Levandwski D.C. (2010). Ausência paterna e suas repercussões para o adolescente: análise da literatura. Psicologia em Revista, 16(2), 295-309.

Silva, E. Z. M. (1999). Paternidade ativa na separação conjugal. São Paulo: Ed. Juarez de Oliveira.

Silva, M. R. (2003). Sentimentos sobre a paternidade e envolvimento de pais que residem e pais que não-residem com seus filhos. (Dissertação de mestrado). Programa de Pós-graduação em Psicologia, Universidade Federal do Rio Grande do Sul, Rio Grande do Sul.

Silva, M. R., \& Piccinini, C. A. (2004). O envolvimento paterno em pais não-residentes: Algumas questões teóricas. Psico, 35, 185-194.

Sisto, F. F. (2001). Dificuldades na aprendizagem em escrita: um instrumento de avaliação (ADAPE). In F.F. Sisto, E. Boruchovitch, L. D. T. Fini, R. P. Brenelli, \& S. C. Martinelli (Orgs.). Dificuldades de aprendizagem no contexto psicopedagógico (pp. 190- 213). Petrópolis: Vozes.

Sitta, E. I., Arakawa, A. M., Caldana, M. L.\& Peres, S. H. C. S. (2010) Contribuição de estudos transversais na área da linguagem com enfoque em afasia. Rev. CEFAC,12(6), 1059-1066.

Torunsky, A. M., \& Lopes, A. M. S. (2012). Influência do bruxismo na ansiedade e aprendizagem em crianças. Clínica e Pesquisa em Odontologia-UNITAU, 4(1), 11-15.

Yaegashi, S. F. R. (1997). O fracasso escolar nas séries iniciais: um estudo com crianças de escolas públicas (Tese de doutorado). Programa de Pós-graduação em Psicologia, Universidade Estadual de Campinas, São Paulo.

Recebido em: 30/04/2013 Primeira decisão editorial em: 18/07/2014 Aceito em: 17/03/2016 